\section{Discrimination and conservation of length*}

\author{
JAMES F. CHRISTIE and DANIEL W. SMOTHERGILL \\ Syracuse University. Syracuse, N.Y. 13210
}

Four-year-olds were Ss in a partial replication of an experiment that had used oddity-problem training to successfully induce conservation of length in 5-year-olds. Training in the present study was successful; a group given knowledge of results on each trial responded to the length cue significantly more often than did an uninformed group. However, neither group evidenced conservation of length on a transfer test. Failure of the present training to induce conservation was predicted by a recent theory of conservation. Hypotheses specifying an identity of processes underlying discrimination and conservation are presented.

A necessary condition for the conservation of, say, length is that the information for length be discriminated from other properties of the stimulus display. For instance, judgments of whether two sticks are equivalent in length must disregard the colors, shapes, end alignments, orientations, etc., of the sticks. Length, an example of what J. J. Gibson (1966) calls a space-distributed stimulus property, must be differentiated from all other coincident, conflicting, and confounding properties if it is to be conserved.

While it seems true on logical grounds that differentiation of length is required for its conservation, it is not so clear whether differentiation is a sufficient condition. The most pertinent research on the question is Gelman's (1967) doctoral thesis (also in Gelman, 1969). Children $(\bar{M}=5$ years, 4 months) identified on pretests as nonconservers of number, length, and amount were presented oddity problems requiring the discrimination of length on half of the trials and number on the remainder. Solution of the problems required that color, alignment, orientation, spacing, and shape be ignored. A group given knowledge of results on each trial

* This report is based on the senior author's VA thesis. The junior author directed the research and prepared this report. We thank Mrs. Lilian Feldman of the Syracuse School District and the teachers and children at the Delaware Baptist Church. This work was partially supported by a contract with the Office of Education, L.S. Department of Health, Education, and Welfare, through the Syracuse Center for Research and Development in Early Childhood Education. Contractors undertaking such work under government sponsorship are encouraged to express freely their professional judgment in the conduct of their work. Points of view or opinions stated do not. therefore, necessarily represent official Office of Education position or policy. The senior author held an NICHD traineeship at the time of this study. Requests for reprints during the 1970-1971 academic vear should be sent to the junior author at the Department of Psychology. Lnjversity of Denver. Denver, Colo. 80210. solved the oddity problems at a high rate and conserved both length and number (and also amount) on posttests. A group not given knowledge of results evidenced little learning and almost no conservation on posttests.

Gelman's results support the hypothesis that differentiation of the property of length is sufficient for conservation of length. Hence, the hypothesis can be formulated that age is relatively unimportant for conservation of length compared to differentiation of the property of length. This hypothesis was tested in the present study by partially replicating Gelman's training procedure with a group of children about a year younger than those used by Gelman.

\section{SUBJECTS AND CONSERVATION TESTS}

Twenty children ranging from 3 years, 6 months to 5 years $(\bar{M}=4$ years, 3 months) were identified as nonconservers of length. Briefly, two sticks of equal lengths were presented parallel to each other with their ends aligned. The children were asked whether the sticks were of the same or different lengths. Correct response was followed by movement of one stick so that the two were no longer end-aligned, and the question was repeated. Five tests of length conservation were given each child, with colors and lengths of sticks varying across tests. The children were asked to explain their answers when the stick ends were not aligned. None of the 20 children classified as nonconservers gave a single conservation response on the pretests. The same procedures were followed on posttests administered to all children the day after training.

\section{TRAINING}

The children were assigned randomly to two equal groups. Both groups received the same 16 six-trial problems requiring response to the odd-length stick. One group (informed) was given knowle dge of results after each trial (and a trinket when correct). While the uther sroup (uninformed) was not given knowledge of results. Ss in the uninformed group were promised a prize at the end if the did well. Each trial consisted of presentation of three sticks. two of which were identical lengths. On half of the trials the question. "Show me the two sticks which are the same length" was asked: on the remaining half the question was. "Show me the stick that 's a different length."

Each of the 16 problems was characterized by one combination of the variables: stick arrangentent. color. diameter. lengths, and shape. There were six trials within each problem. Trial 1 was always set up so that response to either length or end alignment would be correct. On Trials 2-5. length and orientation or end alignment were conflicting cues: responsc to either of the latter would not specify the odd length. On Trial 6 end alignment and orientation did not differentiate among the sticks (cf. Gelman'. 1969. for a fuller description).

\section{RESLiLTS}

Training

A 2 (groups) by 16 (problems) by 6 (trials) ANOVA performed on the number of correct responses found only one significant source of variance: groups $(\mathrm{F}=29.60, \quad \mathrm{~d} f=1 / 18, \quad p<.01) . \quad$ Mean correct responses on 96 trials were 61.5 and 28.9 for the informed and uninformed groups, respectively.

\section{Post test}

None of the children in either group made a single conservation response on the five posttest trials.

\section{DISCUSSION}

Gelman's finding that training $S$ s to discriminate length induced conservation of length was not replicated. There were two major differences between the studies: (1) Gelman's Ss were about 1 year older than the present Ss, and (2) Gelman interspersed number-relevant training trials with length-relevant trials.

The second difference may be crucial. Halford (1970) argues that conservation of length is intrinsically related to conservation of quantity. Indeed, except that number rather than length is the subject of his example, Halford directly anticipated the present results: "Gelman's training procedure encouraged Ss to respond sometimes on the basis of length of the array and sometimes on the basis of number of objects, since length and number problems were interspersed. If Gelman's interpretation is valid, the optimal procedure would seem to be to train Ss exclusively to respond to the number of objects, since length is merely an irrelevant dimension. On the basis of the 
present theory, however, Ss should be trained to respond to changes in both length and number, in order to obtain a maximally complete classification of possibilities [p. 315]." The present results are clearly in keeping with Halford's thesis that training to differentiate only the conservation-relevant cue is insufficient for the acquisition of conservation.

While the results support Halford's position, another interpretation is possible. Although the informed group performed significantly better in training than did the uninformed group, the former may not have fully differentiated the property of length. Over the last half of training, the informed group was correct on $68 \%$ of the trials. The analogous group in Gelman's study responded correctly on $94 \%$ of length-relevant trials over the last half of training. Two hypotheses are suggested by this comparison. First, a common differentiating ability may underlie performance on problems requiring discrimination and conservation of a stimulus property. While fairly reliable discrimination of a stimulus property might occur without its full differentiation, high-level discrimination performance (say, $85 \%$ correct) is coincident with conservation of the stimulus property. Second, the differentiation of stimulus properties which permits their discrimination and conservation normally develops with age.

The two hypotheses taken together imply a stimulus-oriented conception of development (E. J. Gibson, 1969). Further, these hypotheses are contrary to the widely held opinion that the conservations reflect more complex processes than do those involved in discrimination. Rather than minimizing the complexity of conservation, however, the hypotheses suggest that discrimination of a space-distributed stimulus property, such as length, is a developmental acquisition. Research testing both hypotheses is under way in our laboratory.

\section{REFERENCES}

GELMAN, R. S. Conservation, attention, and discrimination. Unpublished doctoral thesis, University of California at Los Angeles, 1967.

GELMAN, R, S, Conservation acquisition: A problem of learning to attend to relevant attributes, Journal of Experimental Child Psychology, 1969, 7, 167-187.

GIBSON, E. J. Principles of perceptual learning and development. New York: Appleton-Century-Crofts, 1969.

GIBSON, J. J. The senses considered as perceptual systems. Boston: Houghton Mifflin, 1966.

HALFORD, G. S. A theory of the acquisition of conservation. Psychological Review, 1970, 77, 302-316.

\title{
The effect on interpersonal attraction of successive and simultaneous presentation of strangers
}

\author{
CHARLES GOUAUX \\ The University of Texas Southwestem Medical School, Dallas, Tex. 75235 \\ and \\ JOHN LAMBERTH \\ University of Oklahoma, Norman, Okla. 73069
}

The experiment was designed to compare two within-Ss designs with the between-groups design generally used in Byrne's interpersonal attraction research. Ss received three agreeing strangers (having 4,8 , or 16 similar attitudes to the Ss) consecutively and evaluated each stranger after reading his attitudes. Other Ss received all three strangers simultaneously and then evaluated each stranger. Whereas $S_{s}$ in the successive-stranger design did not give significantly different attraction scores to the three strangers, for the Ss in the simultaneous-stranger design there was a significant effect attributable to the number of similar attitudes $(\mathrm{p}<.05)$.

In his earliest formulation of his theory of interpersonal attraction, Byrne (1961) proposed that attraction toward a stranger increases as the similarity between the $\mathrm{S}$ and the stranger increases. Extending this conceptualization, Pyrne (1962) suggested that attraction toward a stranger is determined by the number of rewards relative to the number of punishments received from the stranger, but it was realized that this result could be attributed to either the proportion of similar attitudes or the number of similar attitudes. Byrne \& Nelson (1965) pursued this research by testing the hypothesis that attraction toward a stranger is a positive function of the proportion of positive reinforcements received by the $\mathrm{S}$ from the stranger. They employed a 4 by 3 factorial design, with three numbers of positive reinforcements, or similar attitudes between the Ss and the stranger $(4,8$, and 16$)$, and four levels of the proportion of positive reinforcements $(1.00, .67, .50$, and .33). They found, as hypothesized, that the effect due to the number of similar attitudes was nonsignificant, but the proportion effect was highly significant $(p<.001)$. Thus, proportion rather than number of similar attitudes was the effective stimulus variable in this between-groups design. Byrne \& Rhamey (1965) and Byrne \& Clore (in press) further refined mathematically this relationship between interpersonal attraction and proportion of positive reinforcements by including weighting factors for differentially weighted positive and negative reinforcers. The weighting factor was designed to take into consideration the effect of reinforcement magnitude.

In all of this research, between-groups designs were used. No $S$ was required to evaluate more than one stranger. In within-groups designs, there are two ways in which Ss can evaluate more than one stranger in the course of the experiment. With a "successive"-stranger design, a S sees information about and evaluates Stranger 1 prior to receiving any information about Stranger 2, etc. In this case, the first stranger that the Ss evaluate represents a between-groups design. In a "simultane ous"-stranger design, a S receives all of the information from all of the strangers and then proceeds to evaluate the strangers individually. With this design, the $\mathrm{S}$ can make comparisons between the strangers and form his evaluation of each stranger on the basis of these comparisons. Obviously, Ss in a successive design can compare from memory later strangers to earlier ones. The successive-stranger design more nearly approximates the between-groups design employed in earlier work than does the simultaneous-stranger design. Thus, the results of a simultaneous-stranger design may not be totally consistent with between-groups results. Specifically, it is hypothesized that when only the number of similar attitudes is varied, in a simultaneous-stranger design, attraction toward the several strangers is a positive function of the number of attitudes. In a successive-stranger design, 